\title{
LA PREPOSICIÓN A ANTE EL OBJETO DIRECTO EN ITALIANO/LE: ¿FOSILIZACIÓN POR INTERFERENCIA O ERROR DE COMPETENCIA?
}

\author{
Carmen Solsona M artínez \\ U niversidad de Zaragoza
}

RESU MEN: La experiencia docente nos ha demostrado que el error de anteponer la preposición A al objeto directo animado en italiano por parte de alumnos hispanohablantes persiste hasta niveles avanzados de su interlengua. Con el presente trabajo queremos mostrar el grado de incidencia de este error y analizar las causas que lo provocan en un grupo de 48 alumnos españoles de primer nivel de italiano/LE. La prueba que realizaron consistió en la traducción de 20 frases del español al italiano en las cuales aparecía un objeto directo, un objeto indirecto 0 ambos. Nuestro estudio se engloba dentro de una investigación más amplia donde se examinan distintos errores y sus posibles causas en el campo de las ocho preposiciones propias italianas (A, CON, DA, DI, IN, PER, SU , TRA/F RA). M etodológicamente, nos hemos servido del Análisis de Errores (Corder 1967, 1981) y de la Interlengua (Selinker 1969, 1972). Los resultados obtenidos han puesto en evidencia que no se trata exclusivamente de un error interlingüístico (debido a la transferencia de la LM española a la L2 italiana), sino que hay también algunas producciones que demuestran una competencia gramatical deficiente en la propia lengua, lo que lleva a los aprendices a cometer error en la lengua meta.

ABSTRACT: Teaching experience has shown us that the mistake made by Spanish-speaking students of placing the preposition ' $A$ ' before animate direct objects in I talian persists up to advanced levels of their interlanguage. With the present work, we wish to demonstrate the high incidence of this error and its causes in a group of 48 Spanish students from first level of I talian as a foreign language. The test they performed involved the translation of 20 sentences from Spanish to I talian in which a direct object, an indirect object or both appeared. 0 ur study is included within the framework of broader research work in which different mistakes regarding the eight most common I talian prepositions (A, CON, DA, DI, IN, PER, SU , TRA/F RA) and their possible causes are analysed. As far as methodology is concerned, we have used Error Analysis (Corder 1967, 1981) and Interlanguage (Selinker 1969, 1972). The results obtained have revealed that this is not exclusively an interlinguistic error (due to the transfer of the Spanish M T to the Italian L2), but that there are also some productions that demonstrate deficient grammatical competence in the mother tongue, which leads students to make mistakes in the target language.

PALABRAS CLAVE: Análisis de errores y de la Interlengua, preposiciones, italiano/L2.

KEYWORDS: Error Analysis and Interlanguage, prepositions, Italian/L2 


\section{0 bjeto de la investigación}

La preposición es la categoría gramatical que registra - según Katerinov (1975: 37)- un saldo más elevado de errores en alumnos de LM española que aprenden italiano como L $20 \mathrm{LE}$. U no de los errores que quizás presenta mayor tendencia a la fosilización -dentro del campo de las preposiciones italianas para aprendices de LM española- es la anteposición de la preposición a delante del OD animado en italiano. Hablamos de fosilización ${ }^{1}$ porque persiste incluso en niveles avanzados de la interlengua. M ientras en español la preposición a debe anteponerse al OI y precede igualmente al OD si éste es de persona, animal o cosa personificada - no ahondamos por ahora en la complejidad de esta cuestión-, en italiano la preposición a sólo se antepone al primero de los complementos (OI). Es por ello por lo que consideramos que esta disimetría entre el italiano y el español debe explicarse ya desde los comienzos del aprendizaje para, así, evitar -a través de actividades adecuadas- la tendencia natural de anteponer a los objetos directos italianos la preposición -o partícula²- a.

Para abordar el estatuto del error que comentamos en los estudiantes hispanohablantes de italiano, diseñamos un ejercicio que presentamos a éstos consistente en la traducción de veinte frases del español al italiano³ (véase anexo 1).

1. El término fosilización lo definen Richards et al. (1997: 176) como el fenómeno que a veces ocurre en el proceso de adquisición de segundas lenguas y lenguas extranjeras por el que ciertos rasgos incorrectos se convierten en una parte permanente de la manera de hablar o de escribir del aprendiz. El término fue utilizado dentro del ámbito de adquisición de segundas lenguas por Selinker en su artículo "Interlanguage" de 1972. Se trata, para el autor, de un fenómeno que puede afectar a la pronunciación, al vocabulario o a la gramática y es inexistente en la adquisición de una L 1. Selinker notó que la mayoría de los aprendices de L 2 no llegaban a al canzar una buena competencia en la lengua meta debido a interrupciones en su proceso de aprendizaje y se reflejaba en que la interlengua del aprendiz contenía estructuras y reglas distintas a las de la lengua meta. A veces, estas formas fosilizadas (características de estadios de aprendizaje previos) tienden a desaparecer, pero otras son recurrentes en el uso de la lengua (fenómeno que se conoce con el término de backsliding 0 reincidencia).

2. E. Torrego Salcedo (1999: 1779-1805) prefiere hablar de partícula, puesto que la preposición de CD no se comporta como una verdadera preposición. Frente a los complementos preposicionales del verbo, los complementos directos que llevan a se comportan como sintagmas nominales no preposicional es ya que pueden ser sustituidos por pronombres de CD (lo, la, los, las) o pueden convertirse en sujeto de la voz pasiva.

3. Este ejercicio constituye una de las pruebas de una investigación más amplia llevada a cabo de diciembre de 2002 a mayo de 2003 con un grupo de 50 aprendices españoles de italiano como L2. En ella quisimos analizar la interlengua de los aprendices centrándonos en el modo que tenían de adquirir las preposiciones propias italianas, las estrategias de aprendizaje utilizadas, los errores más frecuentes y sus posibles causas. Esta presentación pretende ser un acercamiento a uno de los errores más característicos. 
Todas las frases contenían un complemento u otro (OD, OI) o los dos ${ }^{4}$. Con esta prueba queríamos comprobar la incidencia de esta desviación y la causa que la motivaba ya que, al tratarse de una prueba escrita, el alumno disponía del tiempo suficiente de reflexión para distinguir un complemento de otro y utilizar 0 , por el contrario, dejar de hacerlo, la preposición a. Hemos de aclarar que los estudiantes a la hora de realizar la actividad conocían la regla de que el OD italiano no va precedido de preposición y, de hecho, se les recordó dicha regla antes de comenzar el ejercicio ${ }^{5}$.

N os propusimos intentar discernir, con los resultados obtenidos en la prueba, si se trataba de un error de transferencia o bien, de un problema estrictamente gramatical: el de no saber de qué complemento se trata en su propia lengua, lo que les conduce a error en la L 2 .

\section{Sujetos de la prueba}

Realizaron la prueba 48 alumnos de primer curso de italiano de la Facultad de Filosofía y Letras de la U niversidad de Zaragoza (pertenecientes a distintos estudios universitarios: Filología Hispánica, Inglesa, Francesa, Historia del A rte y de otras facultades) en los primeros 30 minutos de clase el día 5 de mayo de

4. El hecho de que en español el $\mathrm{Cl}$ pueda aparecer acompañado de su correspondiente referente pronominal (le o les) hace que podamos hablar de hasta dos apariciones del mismo complemento indirecto en una misma oración (Le preguntó al guardia dónde estaba la calle). En italiano no encontramos referente pronominal y $\mathrm{Cl}$ en coexistencia (Chiese al vigile dove si trovava la strada/ Gli chiese dove si trovava la strada/*G li chiese al vigile dove...), al menos en el italiano más normativo (sí en el registro hablado o en el neo-standard italiano, véase G. B erruto 1987: 55-103).

5. Decir que el objeto directo en italiano no lleva preposición es una manera muy rápida de querer obviar un fenómeno que, sin duda, precisa de más explicaciones. De hecho, distintos lingüistas italianos se han ocupado del Ilamado accusativo preposizionale (A . Nocentini 1985: 299-311; L. Renzi 1988: 133-5, 155-6, 207-8; M. Berretta 1990: 31-35, A. Zamboni 1991: 51-69). El fenómeno, muy bien caracterizado por M. Berretta (1990: 31-35), consiste en colocar la preposición a delante de nombres o pronombres que se refieren a personas (incluidos los pronombres personales, y siendo la primera persona la más frecuente) en la función de objeto directo. Esta a delante de objeto directo animado, que en español constituye un uso gramatical normal de la a, en italiano es típico del estilo coloquial, caracterizándose también por la posición preferiblemente preverbal, en enunciados enfáticos en los que el elemento que interesa resaltar se coloca delante del verbo (dislocato a sinistra) -especialmente verbos psicológicos como convincere, disturbare, persuadere, preoccupare- y se retoma por medio de un pronombre átono (A me, chi mi consola?). En Italia, el acusativo preposicional se conoce en los dialectos centro-meridionales (en Sicilia, Calabria, Puglia principalmente) y en el italiano regional meridional e insular (Cerdeña, isla de Elba). 
2003, cuando habían recibido 80 horas de clase. Se les dijo que se recogería el ejercicio pero que no tendría calificación.

A principio de curso (septiembre de 2002) se había pasado una encuesta a los alumnos para determinar su perfil. En ella se les preguntaba edad, lengua materna, lenguas extranjeras que conocían, motivación para estudiar italiano y unas preguntas para averiguar su competencia gramatical en español (distinguir verbos transitivos de intransitivos, señalar las distintas categorías gramaticales de una frase e identificar objetos directos e indirectos). R especto de este último ejercicio, las 5 frases propuestas fueron: Le dije la verdad, María compró un ramo de flores a su madre, Os veremos allí, No nos contaron nada más que mentiras, Llamará a los chicos por la tarde. Los resultados menos favorables hacían referencia a la frase 3 (un $43^{\prime} 40 \%$ indicó que os era un 0 I y un $7^{\prime} 55 \%$ no lo identificó de ninguna manera) y a la frase 5 (con un porcentaje de error del 52 ' $83 \%$ que señaló a los chicos como Ol). El resto de las frases mostraron porcentajes de acierto que se movían entre el 85 y el 100\%.

\section{M étodo}

Para nuestro estudio nos hemos basado en los métodos del Análisis de E rrores ( $A E$ ) y de la Interlengua (IL ), teniendo en cuenta también las observaciones del Análisis Contrastivo (A C). Por eso, hablamos de una L 1 o lengua materna del aprendiz (español en nuestro caso), de una L2 o LE (italiano), y de lo que realmente es nuestro objeto de estudio, un sistema aproximado (o interlengua) que no se corresponde con la norma correcta estándar de la $L 2$, pero que refleja la competencia que de ella tiene el aprendiz en ese momento y que, en algunos casos, incluye rasgos de su sistema nativo.

Como hemos indicado, el objetivo de la prueba ha sido valorar la incidencia del error consistente en anteponer la partícula a al OD en italiano y valorar también el grado de discernimiento de los alumnos entre el OD y el OI. En la prueba, todas las frases presentaban un OD, un OI o los dos, ya sea en forma nominal (frase 2: Enrico explicó la lección a su hermano) o pronominal (frase 7: le dijo la verdad). El vocabulario empleado en las frases se ajustaba al que los alumnos conocían a esas alturas del curso pero, aun así, se les proporcionó a pie de página un breve glosario con 13 palabras o expresiones que podían resultar menos conocidas a alguno de los estudiantes.

L os alumnos sabían (o deberían saberlo tras 80 horas de clase) que el OD italiano no lleva delante la preposición a (con la excepción ya indicada en la nota 
5), mientras el español presenta objetos directos precedidos por a (en principio, objetos directos animados: Llamó a P Pietro, Encontré a los chicos, Estoy esperando $\underline{\text { a }}$ P ietro, No puedes obligar a nadie a hacer eso, etc. $)^{6}$. La prueba iba a resultar útil a los alumnos por un lado, porque en caso de duda debían plantearse la diferenciación entre un complemento y otro (bien a través de la pronominalización lo, la, los, las / le, les o a partir de la conversión a pasiva) y, por otro lado, también a nosotros nos podría ayudar a discernir si la colocación de a delante de objetos directos animados en italiano se debía a un error de actuación (el alumno distingue el OD del OI y conoce la regla de omisión de preposición a delante del $O D$, pero por despiste, falta de atención, etc. comete el error de adición de preposición no necesaria) o a un error de competencia gramatical (el alumno tiene dificultad para distinguir un complemento de otro en su lengua materna, lo que le provoca error en la $L 2$ ).

A la dificultad de que la preposición a es compartida en español por el CD y el Cl se añadía otra, que era el leísmo de los estudiantes, ya que en clases anteriores la prueba de pronominalizar el OD había resultado en muchos casos no válida porque escogían los pronombres de OI (le / les) cuando se trataba de objetos con referente personal (*Le llamó, les encontré, le estoy esperando) ${ }^{7}$. De los cuatro tipos de leísmo que distingue Fernández-Ordóñez (1999: 1319), los dos únicos detectados en nuestros alumnos son el referido a un OD animado masculino singular (que, por otro lado, es el leísmo más frecuente y extendido, Vi a J uan y le llamé desde el autobús) y el que hace referencia a un OD también animado pero plural y preferentemente masculino (Encontré a los chicos en la plaza y les invité a tomar un helado). El Ilamado "Ieísmo de cortesía" (como lo ha llamado

6. La presencia de la preposición a delante del OD depende, no obstante, de varios factores (véase E. Torrego Salcedo 1999: 1779-1805): a) el tipo de verbo (con algunos verbos transitivos la anteposición de a implica mayor participación del sujeto en la acción verbal y mayor individuación del complemento Abrazó un niño/ Abrazó a un niño; con verbos télicos -aquellos que describen un proceso que sólo puede darse por realizado con su culminación-: Pedro golpeó a su hermano; con verbos causativos: Hicieron trabajar al obrero sin parar; verbos designativos: Nombraron a J uan director de la empresa); b) la composición interna del complemento directo (con cuantificadores universales la a es obligatoria: Abrazó a todo el mundo, No abrazó a nadie, Abrazó a todos); c) tipo de determinante del objeto directo animado (con determinantes definidos lleva a: Abrazó al niño, *Abrazó el niño); d) tipo de nombre del OD (con nombres propios de persona la preposición es obligatoria: Abrazó a J uan).

7. S. Gili Gaya ([1943] 1989: 233-235) traza un breve pero útil resumen de los hechos lingüísticos referidos al empleo de le como acusativo masculino de persona en las distintas provincias españolas. Para una explicación más detallada y extensa (incluye Hispanoamérica), véase I. Fernández-Ordóñez (1999: 1317-1397). 
A. Lorenzo Ramos 1981), asociado al tratamiento de respeto con usted (Señor director, le llaman por la línea 2), también es frecuente en nuestro grupo aunque no quede reflejado en este ejercicio.

Por ello, suponíamos que las frases que iban a resultar más problemáticas iban a ser aquellas con OD animado a las que llamaremos [grupo A ] y, por tanto, precedidas de la preposición $a^{8}$ (frase 3: Saludé a sus padres y me fui; frase 9: Llamé a J uan desde el autobús; frase 10: Estoy buscando a Pietro desde hace dos horas; frase 11: ¿A quién has invitado a cenar?; frase 12: Abrazó con cariño a su

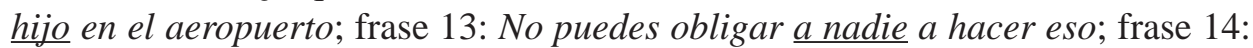
Veo a Pietro todas las mañanas; frase 15: No conocemos a nadie aquí; frase 16: Estoy esperando a Pietro; frase 18: Encontré a los chicos en la plaza; frase 19: Presentó la novia a los amigos; frase 20: Sandro dice que quiere a su novia pero está besando a otra).

En cambio, suponíamos que aquellas frases en las que coaparecían OD y OI -a las que llamaremos [grupo C]- no iban a ser susceptibles de error porque distinguirían fácilmente un complemento de otro -OD no animado de OI animado(frase 1: M i marido cuenta siempre un cuento a los niños antes de dormir; frase 2: Enrico explicó la lección a su hermano; frase 4: Roberto ha mandado ya el libro a su amigo; frase 5: ¿Por qué no pides consejo a tu padre?; frase 7, 2a parte: le dijo la verdad; frase 8: Le preguntó al guardia dónde estaba la calle; frase 17: Envió flores a su novia).

Incluimos, en fin, dos frases [grupo B] que podían ofrecer cierta dificultad: la frase 6 -A los niños les gustan mucho los helados (el verbo gustar aparece en una estructura inacusativa donde el $\mathrm{Ol}$ a los niños expresa el ser animado que experimenta lo expresado por un sujeto inanimado los helados)-, y la frase 7 ( $1^{\text {a }}$ parte) -Llamó por teléfono a Ana (en español Ilamar por tel éfono rige OD pero en italiano correspondería al verbo telefonare que rige $0 \mathrm{I}$, frente a chiamare que regiría un OD).

8. Salvo en la frase 19 que presenta un OD animado pero con la doble particularidad de aparecer junto con un $0 \mathrm{I}$ y de no presentar en español la preposición a porque, como necesita distinguirse de otro complemento con a, prescinde en este caso de la preposición como marca gramatical. 


\section{R esultados}

Los resultados obtenidos han sido en buena medida los esperados aunque, aparte del error de adición de preposición a no necesaria ante objetos directos italianos, nos hemos encontrado con otras desviaciones que analizaremos después. Comencemos por el grupo que creemos encierra mayor dificultad -el que hemos Ilamado grupo A. Estaba compuesto por las frases 3, 9, 10, 11, 12, 13, 14, 15, 16, 18,19 y 20 . Si desglosamos el porcentaje de error (consistente en la colocación de a delante de OD animado) por frases, el resultado es el siguiente 9 .

En la frase 3 (Saludé a sus padres) el acierto es del 44\% (Ho salutato i suoi genitori) y el error del $56 \%$ (*Ho salutato ai / a i / a suoi genitori). La frase 9 (Llamé a J uan) presenta un porcentaje de acierto del $60 \%$ (Ho chiamato J uan) y de error del $40 \%$ (*Ho chiamato a J uan). La frase 10 (E stoy buscando a Pietro) muestra un $75 \%$ de acierto (Sto cercando Pietro) y un $25 \%$ de error (*Sto cercando a Pietro). La frase 11 (¿A quién has invitado a cenar?) presenta un $44 \%$ de acierto (Chi hai invitato a cena?) y un $56 \%$ de error ( ${ }^{*} A$ chi hai invitato a cena?). $\mathrm{L}$ a frase 12 (Abrazó con cariño a su hijo) muestra un 65\% de acierto ( $\mathrm{Ha}$ abbracciato suo figlio) y un $35 \%$ de error ( $* \mathrm{Ha}$ abbracciato a / al suo figlio). La frase 13 (No puedes obligar a nadie a hacer eso) ha mostrado un 73\% de acierto (Non puoi costringere nessuno) y un $27 \%$ de error (*Non puoi costringere a nessuno). En la frase 14 (Veo a Pedro), un $77 \%$ de acierto (Vedo Pietro) y un $23 \%$ de error (*Vedo a P ietro). En la frase 15 (No conocemos a nadie aquí) un $83 \%$ de acierto (Non conosciamo nessuno) y un $17 \%$ de error ( $*$ Non conosciamo a nessuno). En la frase 16 (Estoy esperando a P ietro) un 75\% de acierto (Sto aspettando Pietro) y un $25 \%$ de error (*Sto aspettando a Pietro). En la frase 18 (Encontré a los chicos en la plaza) un $58 \%$ de acierto ( $\mathrm{H}$ o trovato i ragazzi) y un $42 \%$ de error ( $* \mathrm{Ho}$ trovato ai ragazzi). En la frase 19 (P resentó la novia a los amigos) el porcentaje de acierto asciende a un $96 \%$ ( $\mathrm{H}$ a presentato la fidanzata agli amici) y el de error a un escaso 4\% (Ha presentato alla fidanzata agli amici). En la frase 20 (Sandro dice que quiere a su novia pero está besando a otra) un $52 \%$ de acierto (ama la sua fidanzata) y un $48 \%$ de error (*ama a / a la / alla sua fidanzata) en el primer complemento y un $87 \%$ de acierto (sta baciando un'altra) y un $13 \%$ de error en el segundo (*sta baciando a un'altra).

9. Para una visión detallada de los resultados, véanse los cuadros al final del artículo (anexo 2) donde figuran los porcentajes exactos. Para comodidad del lector, ofrecemos en este apartado dichos porcentajes con expresión entera. 
Si nos detenemos en las 13 frases que conforman el grupo que hemos dado en llamar grupo A (OD animado), observamos que la media de error en el conjunto de las frases es de un $31^{\prime} 57 \%$, siendo $56^{\prime} 25 \%$ el porcentaje más alto (correspondiente a las frases 3 y 11) y, el más bajo, de 4'16\% (frase 19). Este porcentaje de error que, a primera vista puede parecer no muy alto, suponemos que en una producción libre (escrita y sobre todo oral donde el nivel de autocorrección es mucho menor) sería todavía mayor. Recordemos que en el encabezamiento del ejercicio figuraba la siguiente instrucción: Traducete in italiano le seguenti frasi facendo attenzione al tipo di complemento utilizzato (complemento oggetto/ complemento indiretto o di termine), lo cual ponía a los aprendices en situación de alerta sobre el tipo de complemento que tenían que traducir y sobre la adición u omisión de preposición a. También es cierto, no obstante, que la traducción es la prueba empírica utilizada en recogida de datos que más favorece la transferencia de la lengua materna. Es, sin embargo, curioso que el porcentaje de error de la frase $19(4 \%)$ es el que más se aleja del resto, quizás porque era la única frase con OD animado sin preposición en español, lo que contribuyó sin duda a una transferencia (en este caso positiva) del español al italiano. El elevado porcentaje de error de la frase 11 (56\%) quizás se deba a la estructura interrogativa de la frase, en la que el OD -en este caso menos explícito- estaba representado por un pronombre interrogativo chi.

E stos resultados se corresponden bastante con los obtenidos en la prueba que se hizo a principio de curso (véase el apartado Sujetos de la prueba). El aprendiz reconocía sin problemas en español el OD inanimado, el OI (sobre todo cuando aparecía junto con un OD), pero demostraba tener más dificultad cuando se trataba de un OD animado (ya sea un sintagma nominal precedido de a o un pronombre átono de los que son comunes para el OD y el OI).

EI grupo que hemos llamado $C$ comprendía las frases con OD inanimado y OI animado: frases 1, 2, 4, 5, 7 (2 - parte), 8 y 17. El error que estas frases podían poner de manifiesto es diferente. Ya no se trata de colocar la preposición a ante los objetos directos (pensábamos que era al go al tamente improbable al tratarse de objetos directos inanimados), sino de omitir la preposición a ante los objetos indirectos (o complementos di termine) o no reconocerlos. La frase 1 (M i marido cuenta siempre un cuento a los niños) presenta un porcentaje de acierto ${ }^{10}$ del $96 \%$

10. Englobamos dentro del acierto incluso aquellos casos en los que la preposición articulada del OI (ai bambini) no era adecuada, bien porque no articulaban la preposición y el artículo determinado (*a i bambini) bien porque el artículo con el que articulaban no era correcto (*agli bambi- 
(M io marito racconta una storia / favola ai bambini) y de error del $4 \%(* M$ io marito racconta una storia i bambini). La frase 2 (Enrico explicó la lección a su hermano) presenta el mismo porcentaje de acierto del $96 \%{ }^{11}$ (Enrico ha spiegato la lezione a suo fratello) y de error del $4 \%$ (*Enrico ha spiegato la lezione suo fratello). La frase 4 (Roberto ha mandado ya el libro a su amigo) muestra un $100 \%$ de aciertos ${ }^{12}$. La frase 5 (¿Por qué no pides consejo a tu padre?) presenta un porcentaje de acierto del $98 \%$ (Perché non chiedi consiglio a tuo padre?) y de error del $2 \%$ (*Perché non chiedi consiglio tuo padre?). En la segunda parte de la frase 7 (... le dijo la verdad), un $96 \%$ de acierto (le ha detto la verità ${ }^{13}$ frente a un $4 \%$ de error (*ha detto la verità). En la frase 8 (Le preguntó al guardia dónde estaba la calle) el acierto es del $96 \%$ (Ha chiesto /Gli ha chiesto al vigile dove si trovava la strada $)^{14}$ y un $4 \%$ de error (*Ha chiesto il vigile...). En la frase 17 (Envió flores a su novia) encontramos un $100 \%$ de acierto ( $\mathrm{Ha}$ inviato dei fiori alla / a la / a sua fidanzata) ${ }^{15}$. EI porcentaje global de error en este grupo ha sido bajo $(2,68 \%)$, siempre inferior al $5 \%$.

Finalmente, el grupo B (compuesto por las frases 6 y 7 - 1 a parte-) ha revelado otros errores que afectan a los complementos analizados. La frase 6 (A los niños les gustan mucho los helados) presenta una dificultad distinta porque ya no se trata de distinguir OD de OI (creíamos que reconocer el Ol era obvio) sino de

ni). De todos modos, esa desviación supone un 14'58 \%, lo que demuestra un manejo todavía inseguro de los artículos determinados italianos y de las contracciones entre preposición y artículo.

11. También en este caso el porcentaje de acierto incluye otras desviaciones que afectan al uso de las preposiciones (en esta frase, el empleo del artículo determinado delante de un posesivo con un nombre de familia en singular al suo fratello).

12. También en esta frase ha habido desviaciones (un $20^{\prime} 83 \%$ ) debidas bien a la no articulación entre preposición y artículo (*a il suo amico), bien a la eliminación -quizás por hipercorrección- del artículo determinado (*a suo amico).

13. Hemos incluido en los aciertos las frases en las que el pronombre átono de $0 \mathrm{I}$ no era le (que sería el correcto al tratarse de un OI femenino singular) sino también gli.

14. Hemos incluido en el porcentaje de acierto los casos de duplicación del OI con un pronombre átono. Colocamos intencionadamente dos frases muy parecidas (5 y 8), con la salvedad de que en la frase 8 habíamos duplicado el OI con un pronombre átono (Le preguntó al guardia dónde estaba la calle) y en la 5 no (¿Por qué no pides consejo a tu padre?). Cuando en la frase para traducir aparecía el pronombre, un $60^{\prime} 42 \%$ de los alumnos lo ha reproducido en italiano (aun cuando esta lengua rechaza los pleonasmos pronominales en el OI) mientras que, cuando no aparecía en español, tampoco ha sido traducido al italiano ( $0 \%$ de casos de duplicación). Estos resultados ponen de manifiesto que la causa del error es claramente de natural eza interlingüística (transferencia del español al italiano).

15. Hemos incluido en los aciertos las contracciones incorrectas entre preposición y artículo determinado 0 la ausencia de éste. 
reconocer el $\mathrm{OI}$ en una posición que no es la habitual en la estructura de la frase en español (SVO). La frase 6 ha mostrado un porcentaje de acierto del 85\% ( $\mathrm{Ai}$ bambini piacciono molto i gelati $)^{16}$ y un porcentaje de error del $15 \%$, que se ha reflejado en la confusión del 0 I con el sujeto (*I bambini piacciono molto i gelati). La primera parte de la frase 7 (Llamó por teléfono a A na....) ${ }^{17}$ ha presentado un $37 \%$ de acierto ( $\mathrm{H}$ a tel efonato a / ad Anna...) y un $63 \%$ de error ( $* \mathrm{H}$ a telefonato Anna). El error, a pesar de que podía deberse a la confusión del OI con el OD dejando por ello sin preposición al complemento italiano, consiste en cambio para el que lee- en creer que dicho complemento (en español a Ana y en italiano Anna) es sujeto (Ha llamado por teléfono Ana).

\section{Conclusiones}

B uena parte de los errores registrados son de natural eza interlingüística y se deben a transferencias lingüísticas del español al italiano. Nos referimos, por ejemplo, a los casos de duplicación del OI a través de pronombres átonos. Es muy comprensible que así sea, puesto que, como señala Giacalone R amat en H. D ulay et al. ([1982]1985: 20), la interferencia actúa como estrategia de aprendizaje en la formación de hipótesis sobre la estructura de la lengua meta, jugando un importante papel en la configuración de la interlengua. Este hecho es todavía más evidente en lenguas tipológicamente cercanas entre sí, como el español y el italiano ${ }^{18}$ aunque, en algunos casos como los mencionados, las frases erróneas sean resultado de hipótesis de los aprendices que se han demostrado ineficaces.

En el caso de adición de la preposición a delante de OD animado podemos hablar también de interferencia lingüística con el español, aunque en algunos casos reconocemos que es difícil afirmar con seguridad que sea ésta su única

16. Hemos incluido en los aciertos los casos de duplicación del OI con un pronombre átono. Esta desviación ha supuesto un 58' $39 \%$. En español, se trata de uno de los pocos casos en los que la presencia del pronombre átono de 0 I es obligatoria: cuando el constituyente prepositivo aparece en primer término. V éase S. Gutiérrez Ordóñez (1999: 1871).

17. Hemos de decir que en el pequeño glosario a pie de página con la traducción de algunas palabras y expresiones habíamos incluido "Ilamar por teléfono" = telefonare para evitar que usasen el verbo chiamare, que rige OD.

18. S. Schmid (1994: 109-120) señala que la estrategia de la congruencia (descubrimiento de elementos y estructuras parecidos en distintos niveles del sistema) es ampliamente utilizada en los primeros estadios de aprendizaje de la $\mathrm{L} 2$. 
causa, ya que al gunas de las soluciones dadas en el grupo A ponen en evidencia que los alumnos no sabían si se trataba de un complemento u otro en su LM. Hemos comprobado que al gunos verbos como baciare, aspettare, conoscere eran más fáciles que otros como salutare, invitare, incontrare, amare. Por "fácil" o difícil" queremos expresar que hay verbos en los que el aprendiz reconoce un OD evidente, mientras que en otros opina, equivocadamente, que se trata de OI. EI error puede deberse a que las pruebas de pronominalización o de conversión a pasiva en estos casos sean menos convincentes 0 a la propia estructura de la frase (como la frase 11, que es una frase interrogativa en la que el pronombre chi es el OD).

Para evitar los casos de interferencia, la práctica reiterada de ejercicios puede resultar muy útil ${ }^{19}$ (querer, odiar, saludar, Ilamar, buscar, conocer, esperar, recibir, acompañar, ayudar, mirar, ver, encontrar a alguien, a María, a Pedro, a los niños, quererla, odiarlo, saludarlas, llamarlos ... / amare, odiare, salutare, chiamare, cercare, conoscere, aspettare, ricevere, accompagnare, aiutare, guardare, trovare o incontrare qualcuno, Maria, Pietro, i bambini, amarla, odiarlo, salutarle, chiamarli).

En los casos de competencia gramatical deficiente (confusión de OD y OI) convendría dedicar un tiempo a solucionar este problema, explicando la diferencia entre verbos transitivos e intransitivos, evidenciando la tendencia leísta ${ }^{20}$ que confunde al gunas veces a la hora de reconocer de qué complemento se trata en español, y haciendo ver a nuestros alumnos que, en algunos casos como los presentados, una competencia gramatical deficiente en la L M ocasiona serios proble-

19. El significativo porcentaje de error en el apartado de contracciones incorrectas (ya sea por elección incorrecta del artículo determinado, ya sea por el modo de llevar a cabo la articulación entre preposición y artículo determinado) también refleja la necesidad de insistir a través de ejercicios adecuados en este terreno que separa a la $L 1$ de la $L 2$, ya que en español sólo tenemos dos ejemplos de articulación entre la preposición y el artículo determinado (al y del) frente a las 35 posibilidades que ofrece el italiano.

20. Sería útil realizar el mismo ejercicio con aprendices españoles de italiano de otras regiones españolas para ver qué relación existe entre población leísta y colocación de a delante de OD animado en italiano. A raíz de los resultados obtenidos, consideramos que otro ejercicio que podría resultar útil sería la traducción del español al italiano de oraciones con verbos que admiten OD preposicional en español (y que señala Torrego Salcedo: verbos de afección, suasivos, transitivos, designativos, télicos, causativos) para ver cuáles de esas categorías registran más casos de anteposición de a a los objetos directos italianos y cuáles menos. 
mas en el aprendizaje de una $L 2^{21}$. Los casos de omisión de la preposición a delante de 0 I creemos que se deben principalmente a hipercorrecciones.

\section{Bibliografía}

A RCE, J. et al. Italiano y español. Estudios lingüísticos. Sevilla: Publicaciones de la Universidad de Sevilla, 1984.

ARRIAGA FLOREZ, M . et al. (coords.) Italia-E spaña-E uropa: Literaturas comparadas, Tradiciones y Traducciones, A ctas del XI Congreso Internacional de la Sociedad Española de Italianistas. Sevilla: A rcibel, 2005.

BERRETTA, M. "E a me chi mi consola?". Italiano e Oltre, 1990, 1 (genn.febb.), pp. 31-35.

BERRUTO, G. Sociolinguistica dell'italiano contemporaneo. Roma: La Nuova Italia, 1987.

BOSQUE, I. y V. DEMONTE (eds.) Gramatica Descriptiva de la Lengua Española. M adrid: Espasa Cal pe, 1999.

CALVI, M a. V. Didattica di lingue affini. Spagnolo e italiano. M ilano: Guerini, 1995.

- "A prendizaje de lenguas afines: español e italiano" en SIERRA MARTÍNEZ, F. y HER NÁ NDEZ GONZÁ LEZ, C. (eds.), 1999, pp. 23-45.

CORDER, S. P. "The significance of learner's errors". International Review of Applied Linguistics, 1967, 5, pp. 161-170.

- Introducing A pplied Linguistics. Harmondsworth: Penguin B ooks L td., 1973.

- Error analysis and interlanguage. Oxford: Oxford U niversity Press, 1981.

DULAY, H., BURT, M. y KRASHEN, S. Language Two. New York: Oxford University Press, 1982. Versión italiana: La seconda lingua (ed. a cura di A. Giacalone Ramat). B ologna: II M ulino, 1985.

FERNÁNDEZ LÓPEZ, C. Las preposiciones. Valores y usos. Salamanca: Ediciones del Colegio de España, 1999.

FERNÁNDEZ-ORDÓÑEZ, I. "Leísmo, laísmo y loísmo" en BOSQUE y DEM ONTE (eds.), 1999, vol. 1, cap. 21, pp. 1317-1397.

21. Como señala Carrera en J. A rce et al. (1984: 209), el hablante español se inclina en muchos casos a discriminar el empleo de los pronombres lo y le no respecto de su función (OD u OI) -como sería lo correcto- sino según el criterio de referencia no personal / personal. Este hecho es un ejemplo más, junto con la a ante OD animado, que revela cómo el español (en comparación con el italiano) tiende a destacar la persona en la sintaxis y dicha tendencia, como hemos visto, se transfiere muchas veces a la $\mathrm{L} 2$. 
GILI GAYA, S. 1943, Curso superior de sintaxis española. B arcelona: Biblograf, 1989.

GUTIÉRREZ ORDÓÑEZ, S. "Los dativos" en BOSQUE y DEMONTE (eds.), 1999, vol. 2, cap. 30, pp. 1855-1931.

KATERINOV, K. "L'analisi contrastiva e l'analisi degli errori di lingua applicate all'insegnamento dell'italiano a stranieri". Rassegna Italiana di Linguistica Applicata, 1975, VII, 2-3, Roma: Bulzoni, pp. 17-69.

LORENZO RAMOS, A. "Algunos datos sobre el leísmo en el español de Canarias" en ALVAR, M. (coord.). I Simposio Internacional de Lengua Española. L as Palmas: Ediciones del Excmo. Cabildo Insular de Gran Canaria, 1981, pp. 175-180.

NOCENTINI, A. "Sulla genesi dell'oggetto preposizionale nelle lingue romanze". Studi Linguistici e Filologici per Carlo Alberto Mastrelli. Pisa: Pacini, 1985, pp. 299-311.

RENZI, L. (ed.) 1988, Grande Grammatica di consultazione (volume I: L a frase. I sintagmi nominale e preposizionale). Bologna: il Mulino, 1991.

RICHARDS, J. C., J. PLATT y H. PLATT. Diccionario de lingüística aplicada y enseñanza de lenguas. Barcelona: A riel, 1997 (versión española de M uñoz Lahoz, C. y C. Pérez Vidal de la versión original en inglés de 1992: Longman Dictionary of Language Teaching and Applied Linguistics. London: Longman).

SCHMID, S. L'italiano degli spagnoli. Interlingue di immigrati nella Svizzera tedesca. M ilano: FrancoA ngeli, 1994.

SELINKER, L. "L anguage transfer". G eneral Linguistics, 1969, 9, pp. 67-92.

- "Interlanguage". International Review of Applied Linguistics, 1972, 10, pp. 209-231.

SIERRA MARTÍNEZ, F. y HERNÁNDEZ GONZÁ LEZ, C. (eds.) Las lenguas en la Europa Comunitaria III. Colección Diálogos Hispánicos, 23, A msterdam-A tlanta: Rodopi, 1999.

SOLSONA MARTÍNEZ, C. "Asimilación de las preposiciones propias italianas por parte de aprendices hispanohablantes de italiano/L E: estrategias, errores y posibles causas", en ARRIA GA FLÓREZ, M. et al. (coords.), 2005, pp. 705716.

TORREGO SA LCEDO, E. "EI complemento directo preposicional" en BOSQUE y DEM ONTE (eds.) 1999, vol. 2, cap. 28, pp. 1779-1805.

ZAMBONI, A. "Postille alla discussione sull'accusativo preposizionale". Quaderni dell'Istituto di G lottologia, 1991, 3, Università di Chieti, pp. 51-69. 


\section{Anexo 1}

Traducete in italiano le seguenti frasi facendo attenzione al tipo di complemento utilizzato (complemento oggetto/ complemento indiretto o di termine).

1. M i marido cuenta siempre un cuento a los niños antes de dormir.

2. Enrico explicó la lección a su hermano.

3. Saludé a sus padres y me fui.

4. Roberto ha mandado ya el libro a su amigo.

5. ¿Por qué no pides consejo a tu padre?

6. A los niños les gustan mucho los helados.

7. Llamó por teléfono a A na y le dijo la verdad.

8. Le preguntó al guardia dónde estaba la calle.

9. Llamó a J uan desde el autobús.

10. Estoy buscando a Pietro desde hace dos horas.

11. ¿A quién has invitado a cenar?

12. A brazó con cariño a su hijo en el aeropuerto.

13. No puedes obligar a nadie a hacer eso.

14. Veo a Pietro todas las mañanas.

15. No conocemos a nadie aquí.

16. Estoy esperando a Pietro.

17. Envió flores a su novia.

18. Encontré a los chicos en la plaza.

19. Presentó la novia a los amigos.

20. Sandro dice que quiere a su novia pero está besando a otra.

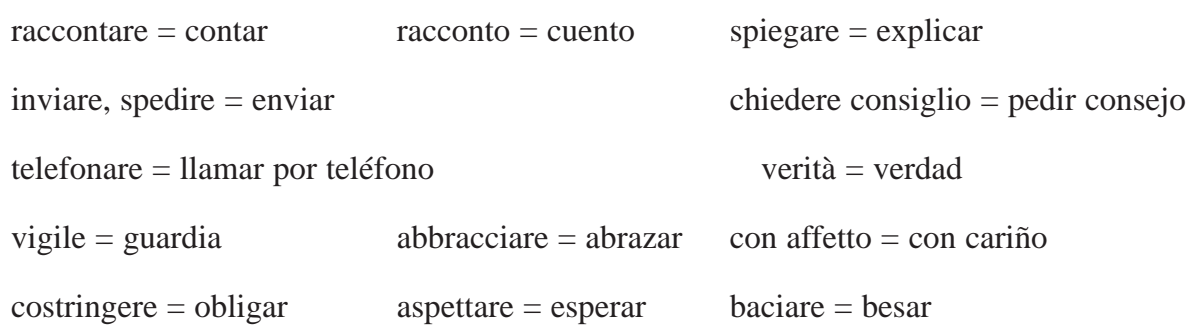




\section{Anexo 2}

Cuadro 1 (Porcentajes de acierto y error en Grupo A)

\begin{tabular}{|l|r|r|}
\cline { 2 - 3 } \multicolumn{1}{c|}{} & Acierto & Error \\
\hline frase 11 & $43,75 \%$ & $56,25 \%$ \\
frase 3 & $43,75 \%$ & $56,25 \%$ \\
frase 20 (1) & $52,08 \%$ & $47,92 \%$ \\
frase 18 & $58,33 \%$ & $41,67 \%$ \\
frase 9 & $60,42 \%$ & $39,58 \%$ \\
frase 12 & $64,58 \%$ & $35,42 \%$ \\
frase 13 & $72,91 \%$ & $27,09 \%$ \\
frase 10 & $75,00 \%$ & $25,00 \%$ \\
frase 16 & $75,00 \%$ & $25,00 \%$ \\
frase 14 & $77,09 \%$ & $22,91 \%$ \\
frase 15 & $83,33 \%$ & $16,67 \%$ \\
frase 20 (2-) & $87,50 \%$ & $12,50 \%$ \\
frase 19 & $95,83 \%$ & $4,17 \%$ \\
\hline Media & $\mathbf{6 8 , 4 3 \%}$ & $\mathbf{3 1 , 5 7 \%}$ \\
\hline
\end{tabular}

\section{Cuadro 2 (Porcentajes de acierto y error en G rupo B)}

\begin{tabular}{|l|r|r|}
\cline { 2 - 3 } \multicolumn{1}{c|}{} & Acierto & E rror \\
\hline frase 7 (1)ㅡㄹ & $37,50 \%$ & $62,50 \%$ \\
frase 6 & $85,39 \%$ & $14,61 \%$ \\
\hline Media & $\mathbf{6 1 , 4 5 \%}$ & $\mathbf{3 8 , 5 6 \%}$ \\
\hline
\end{tabular}

Cuadro 3 (Porcentajes de acierto y error en Grupo C)

\begin{tabular}{|l|r|r|}
\cline { 2 - 3 } \multicolumn{1}{c|}{} & Acierto & E rror \\
\hline frase 1 & $95,83 \%$ & $4,17 \%$ \\
frase 8 & $95,83 \%$ & $4,17 \%$ \\
frase 2 & $95,83 \%$ & $4,17 \%$ \\
frase 7 (2a) & $95,83 \%$ & $4,17 \%$ \\
frase 5 & $97,91 \%$ & $2,09 \%$ \\
frase 4 & $100,00 \%$ & $0,00 \%$ \\
frase 17 & $100,00 \%$ & $0,00 \%$ \\
\hline Media & $\mathbf{9 7 , 3 2} \%$ & $\mathbf{2 , 6 8 \%}$ \\
\hline
\end{tabular}

